\title{
Estudio arqueomineralógico de las menas de cobre prehispánicas en Collahuasi, norte de Chile
}

\section{Archaeo-mineralogical study of Prehispanic copper ores in Collahuasi, northern Chile}

\author{
Pía Sapiains ${ }^{1}$ https://orcid.org/0000-0001-8849-4620 \\ Andrew Menzies ${ }^{2}$ https://orcid.org/0000-0003-0343-5758 \\ Valentina Figueroa ${ }^{3}$ https://orcid.org/0000-0001-7485-7013 \\ Hans-Gerhard Wilke ${ }^{4}$ https://orcid.org/0000-0003-1021-1242 \\ Benoit Mille ${ }^{5}$ https://orcid.org/0000-0003-0151-265X \\ Diego Salazar ${ }^{6}$ https://orcid.org/0000-0002-1438-0839 \\ José Berenguer ${ }^{7}$ https://orcid.org/0000-0003-1319-1654 \\ ${ }^{1}$ Doctorado en Ciencias mención Geología, Departamento de Ciencias Geológicas, \\ Universidad Católica del Norte, Antofagasta, CHILE. Email: psg004@ucn.cl \\ ${ }^{2}$ Bruker Nano GmbH, Berlín, ALEMANIA. Email: andrew.menzies@bruker.com \\ ${ }^{3}$ Instituto de Investigaciones Arqueológicas y Museo (IIAM), Universidad Católica del Norte, San Pedro de \\ Atacama, CHILE. Email: vfigueroa@ucn.cl \\ ${ }^{4}$ Anqa, servicios geológicos y paleontológicos, Antofagasta, CHILE. \\ Email: hgbw1953@gmail.com \\ ${ }^{5}$ Centre de Recherche et de Restauration des Musées de France, Paris, Francia \& UMR 7055 Préhistoire et \\ Technologie, Nanterre, FRANCIA. Email: benoit.mille@culture.gouv.fr \\ ${ }^{6}$ Departamento de Antropología, Universidad de Chile, Santiago, CHILE. \\ Email: dsalazar@uchile.cl \\ ${ }^{7}$ Museo Chileno de Arte Precolombino, Santiago, CHILE. \\ Email: jberenguer@museoprecolombino.cl
}

\section{Resumen}

El Norte de Chile es conocido por la importante explotación cuprífera contemporánea. Las evidencias arqueológicas indican que tal explotación no es un fenómeno moderno, sino que ha existido en el Norte Grande desde al menos el Período Arcaico Tardío. Para identificar las materias primas minerales usadas en los procesos minero-metalúrgicos prehispánicos, hemos desarrollado un nuevo protocolo analítico basado en la mineralogía automatizada (QEMSCAN). Las muestras provienen del distrito minero-metalúrgico prehispánico de Collahuasi, ubicado en la actual región de Tarapacá. A partir de este estudio, se determinó que la mineralogía de las menas utilizadas en las fundiciones metalúrgicas de los sitios del Distrito Collahuasi corresponde a brocantita, malaquita y crisocola; a su vez, se ha podido poner en evidencia cuál fue el "metal Collahuasi" que circuló en tiempos prehispánicos tardíos.

Palabras clave: cobre, arqueomineralogía, arqueometalurgia, Collahuasi, QEMSCAN.

\begin{abstract}
Northern Chile is known for its large-scale contemporary copper exploitation. Archaeological evidence indicates that such exploitation is not only a modern occurrence but has been ongoing since at least the Late Archaic Period. In order to identify the raw mineral materials used in pre-Hispanic mining and metallurgical production processes we performed an automated mineralogy analysis (QEMSCAN). The samples are from the Collahuasi miningmetallurgical district, in the Tarapacá region. It was determined that the primary mineralogy of the ore in the pre-Hispanic smelting sites of the Collahuasi District was brochantite, malachite and chrysocolla, and in turn, it has been possible to determine what the "Collahuasi metal" was, that circulated in late prehispanic times.
\end{abstract}

Keywords: copper, archaeomineralogy, archaeometallurgy, Collahuasi, QEMSCAN.

Recibido: 1 noviembre 2017. Aceptado: 7 enero 2020 


\section{Introducción}

En el norte de Chile se encuentra una de las franjas metalogénicas de cobre más ricas del mundo (Sillitoe, 2005), existiendo en la actualidad un número importante de las minas más grandes del planeta, como Chuquicamata y Escondida. Sin embargo, es conocido que la explotación de minerales de cobre no es un fenómeno moderno y que los antiguos habitantes utilizaron estos recursos minerales en diferentes producciones especializadas, a saber, la lapidaria (Rees, 1999), pigmentaria (Salazar et al., 2010a; Figueroa, Salazar, Salinas, Núñez-Regueiro y Manríquez, 2013; Sepúlveda, Figueroa y PagésCamagna, 2013; Sepúlveda, Figueroa y Cárcamo, 2014), y en la metalurgia (Salazar, Figueroa, Mille, Morata y Salinas, 2010b; Salazar et al., 2011, Figueroa et al., 2018), milenios antes.

El objetivo principal de este estudio es comprender el sistema de producción minera y metalúrgica durante el Período Intermedio Tardío (850-1470 DC) y Período Tardío (1470-1536 DC) en el Distrito Collahuasi, contribuyendo así a nuestra comprensión de la variabilidad y de las transformaciones de la metalurgia andina a través del tiempo (Figueroa et al., 2018). Desde esta perspectiva, es de central importancia comprender la organización social de las operaciones minero-metalúrgicas y de las tecnologías disponibles. En este trabajo nos centraremos en los minerales de cobre seleccionados por mineros y metalurgistas prehispánicos para ser fundidos a través de un proceso metalúrgico muy específico y particular (Mille et al., 2013), empleando un sistema que utilizó el viento para transformar cobre mineral en cobre metálico, llegando a los $1200{ }^{\circ} \mathrm{C}$ por un lapso que se mantuvo, ayudado por combustibles locales como la queñoa (Polylepis tarapacana Phil.), la tola (Parastrephia quadrangularis) y la llareta (Azorella compacta) para alcanzar la fundición (Figueroa et al., 2018).

Las muestras provienen de sitios arqueológicos prehispánicos situados en el Distrito Collahuasi (región de Tarapacá), ubicados a $4500 \mathrm{msnm}$, entre 6 y 9 $\mathrm{km}$ de los yacimientos Rosario y Ujina, respectivamente (Figura 1). Estos forman parte de los pocos sitios metalúrgicos prehispánicos bien conservados en el norte de Chile, por cuanto ha existido una explotación diacrónica en los principales distritos minero-metalúrgicos, la cual ha aumentado considerablemente de escala en los últimos 30 años, teniendo directas repercusiones materiales en las explotaciones más antiguas (Salazar, 2010). En este trabajo, se presentan los resultados mineralógicos de muestras tomadas de un sitio residencial doméstico de metalurgistas (Collahuasi 37) y de dos sitios de producción metalúrgica (Ujina 8 y 10) (Figura 1). Los sitios mencionados han sido descritos por Romero y Briones (1999); Berenguer, Sanhueza y Cáceres (2011); Salazar et al. (2013a); Figueroa (2015, 2016), y en las últimas campañas de terreno se han registrado tres nuevos sitios metalúrgicos (Ujina 9, 11 y 12) (Figueroa et al., 2018).

El Análisis Mineralógico Automatizado QEMSCAN (Quantitative Evaluation of Materials by Scanning Electron Microscopy) es un método común utilizado para describir la mineralogía de una muestra mineral, especialmente relacionado con la mineralogía de cobre (Menzies, Campos, Hernández, Sola y Riquelme, 2015a; Menzies et al. 2015b; Sapiains, 2016). La mineralogía automatizada se ha aplicado ampliamente en la industria minera desde hace más de 20 años, especialmente en el norte de Chile, en varios depósitos de cobre (Menzies et al., 2015a), pero rara vez en contextos arqueológicos. Recientemente, se ha utilizado para análisis estratigráficos del sedimento de sitios arqueológicos australianos (Ward et al., 2018), y en Chile se ha realizado este análisis en términos prospectivos en cerámica prehispánica (Menzies, Uribe, Erazo, Ossandón y Fonseca, 2015c). Cabe señalar que se trata de una técnica no destructiva, pero que requiere pulir una superficie de la muestra para facilitar el análisis, por lo que no puede ser aplicada en muestras arqueológicas cuya conservación es fundamental, ya que se modifica el estado original y/o museográfico de la pieza. Sin embargo, la mineralogía automatizada además de determinar los minerales presentes en la muestra, a través de los mapas mineralógicos brinda información acerca de los porcentajes de cada uno de los minerales identificados, y cómo se distribuyen en la muestra, incluso pudiéndose observar sus texturas y elementos trazas incluidos en las especies minerales, lo que representa una ventaja adicional en comparación con los métodos clásicamente usados en arqueometría (Fluorescencia de Rayos X, Espectroscopia RAMAN). 


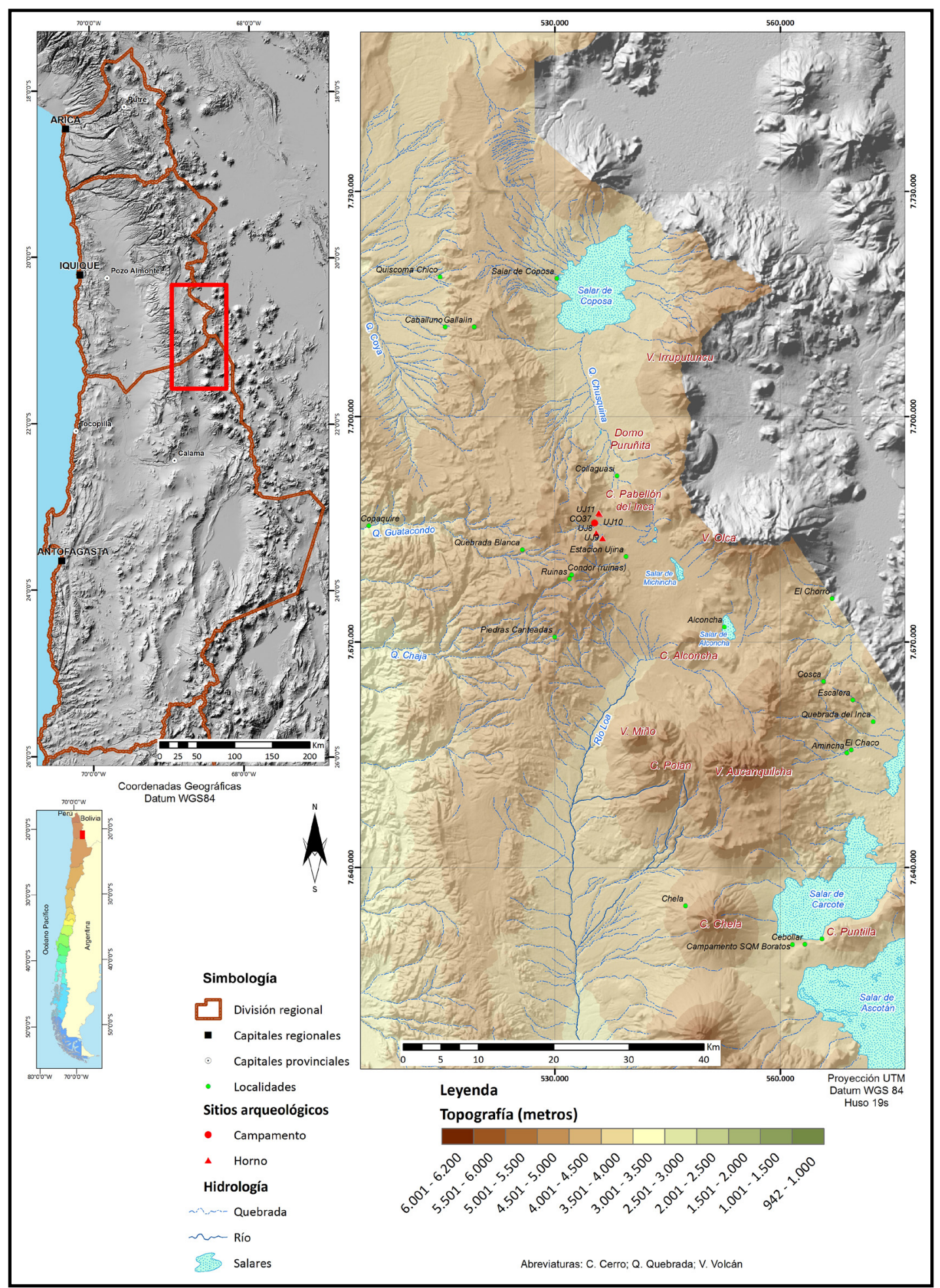

Figura 1. Ubicación de los sitios arqueológicos en el Distrito Collahuasi. 


\section{Selección y preparación de las muestras}

Para este estudio, se seleccionaron 40 fragmentos de roca en la primera campaña de terreno realizada en enero de 2015, provenientes de tres sitios arqueológicos que muestran evidencia de actividades metalúrgicas indígenas, específicamente correspondientes a los sitios Collahuasi 37, Ujina 8 y Ujina 10. Los sitios Ujina 8 y 10 tienen dos características importantes: una alta concentración de escorias metalúrgicas en la superficie y numerosos hornos metalúrgicos confeccionados con piedras (Salazar et al., 2013a; Figueroa et al., 2018). Estas estructuras pirometalúrgicas fueron construidas con características comunes, particularmente una gran longitud (4 a $10 \mathrm{~m}$ ), una pared posterior de una altura no superior a $1 \mathrm{~m}$ (Figueroa et al., 2018) y orientadas perpendicularmente a la dirección de los vientos dominantes para alcanzar las temperaturas adecuadas, llegando a los $1200{ }^{\circ} \mathrm{C}$, para llevar a cabo la fundición del cobre (Mille et al., 2013, Figueroa et al., 2018).

Se utilizaron dos criterios para la recolección de muestras: i) que la muestra de mano tuviera evidente mineralización de oxidados de cobre (estas fueron seleccionadas en terreno por los investigadores con ayuda de una lupa geológica), y; ii) que las muestras se encontraran en los sitios arqueológicos mencionados. Específicamente, en Collahuasi 37 se tomaron del interior de las estructuras pircadas y en los sitios Ujina 8 y 10 a no más de $0,5 \mathrm{~m}$ de las estructuras pirometalúrgicas. Esto maximizó la probabilidad de que estas muestras tomadas de la superficie de los sitios arqueológicos estén directamente relacionadas con las muestras de mineral primario utilizadas en estos sitios. De las 40 muestras recolectadas, solo 20 fueron analizadas con mineralogía automatizada. El número seleccionado de muestras es proporcional al número total de muestras de cada sitio, seleccionando $-50 \%$ de cada sitio, esto principalmente por razones restrictivas del método; algunas muestras no superaban el tamańo requerido para obtener una sección pulida. Se proporciona información de las muestras en la Tabla 1 y en la Figura 2.

\section{Metodología analítica}

Los análisis mineralógicos automatizados QEMSCAN (Quantitative Evaluation of Materials by Scanning Electron Microscopy) miden la variabilidad mineralógica de una muestra, basada en la geoquímica a escala micrométrica. Dicha información puede corroborar o descartar la información proporcionada por la microscopía tradicional, así como cuantificar con precisión la mineralogía modal y la conducta elemental. En la última década, la mineralogía automatizada (QEMSCAN) se ha aplicado rutinariamente para ayudar a entender los minerales de cobre, y así contribuir a mejorar el procesamiento metalúrgico de este metal (Menzies et al., 2015a; Campos et al., 2015).

Las muestras analizadas midieron entre 1 y $5 \mathrm{~cm}$ y fueron montadas en briquetas circulares de resina epóxica. Luego las briquetas fueron curadas, pulidas y recubiertas en carbón, antes de llevar a cabo el análisis mineralógico automatizado.

Los análisis se realizaron mediante QEMSCAN modelo E430, que se basa en un microscopio electrónico de barrido (SEM) ZEISS EVO 50 combinado con detectores espectrales de energía dispersiva (EDS) Bruker Series 4. El análisis de rutina se realiza con un tamańo de punto de menos de $1 \mu \mathrm{m}$, con un voltaje operativo de $25 \mathrm{kV}$ y un haz de corriente de $5 \mathrm{nA}$. Se realizaron mil conteos estándar por punto con un límite de detección de aproximadamente $2 \%$ en peso por elemento para cada punto de clasificación mineral. Las mediciones se realizaron con iMeasure v5.3.2 y la reducción de datos mediante iDiscover v5.3.2. Las muestras se analizaron en el modo Fieldscan en un tamańo de campo de 1500 $\mu \mathrm{m}$ (aumento aproximado de 50x) o $2500 \mu \mathrm{m}$ (aumento aproximado de 30x). Se realizaron múltiples análisis a diferentes resoluciones de separación de pixeles, generalmente 10,5 o $2 \mu \mathrm{m}$ para determinar la mineralogía modal, así como para observar texturas a escala fina y mineralización. Los niveles de electrones retrodispersados se calibraron de 0-255, donde Cuarzo $=42$, Cobre $=130$ y Oro $=232(\mathrm{Fi}-$ gura 3). 
Tabla 1. Localización en coordenadas UTM de cada muestra analizada con mineralogía automatizada. Datum WGS 84 UTM 19S. Tomados con un aparato modelo etrex 10, marca Garmin. Error de precisión 3 metros.

\begin{tabular}{|c|c|c|c|c|}
\hline Código de muestra & $\begin{array}{c}\text { Coordenada UTM } \\
(\mathrm{mN})\end{array}$ & $\begin{array}{c}\text { Coordenada UTM } \\
(\mathrm{mE})\end{array}$ & Sitio arqueológico & $\begin{array}{r}\text { Altura } \\
(\mathbf{m s n m})\end{array}$ \\
\hline UJ8-m8-a & 7.684 .416 & 535.423 & Ujina 8 & 4.385 \\
\hline UJ8-m8-c & 7.684 .416 & 535.423 & Ujina 8 & 4.385 \\
\hline UJ8-m6-a & 7.684 .523 & 535.489 & Ujina 8 & 4.386 \\
\hline UJ8-m6-b & 7.684 .523 & 535.489 & Ujina 8 & 4.386 \\
\hline UJ8-m6-c & 7.684 .523 & 535.489 & Ujina 8 & 4.386 \\
\hline UJ8-m6-d & 7.684 .523 & 535.489 & Ujina 8 & 4.386 \\
\hline UJ8-m5-a & 7.684 .585 & 535.488 & Ujina 8 & 4.378 \\
\hline Co37-m13-b & 7.685 .832 & 535.318 & Collahuasi 37 & 4.302 \\
\hline Co37-m12-b & 7.685 .865 & 535.253 & Collahuasi 37 & 4.312 \\
\hline Co37-m11-b & 7.685 .846 & 535.298 & Collahuasi 37 & 4.306 \\
\hline Co37-m10-b & 7.685 .846 & 535.298 & Collahuasi 37 & 4.306 \\
\hline Co37-m10-e & 7.685 .846 & 535.298 & Collahuasi 37 & 4.306 \\
\hline Co37-m10-g & 7.685 .846 & 535.298 & Collahuasi 37 & 4.306 \\
\hline Co37-m10-i & 7.685 .846 & 535.298 & Collahuasi 37 & 4.306 \\
\hline Co37-m10-j & 7.685 .846 & 535.298 & Collahuasi 37 & 4.306 \\
\hline Co37-m9-a & 7.685 .856 & 535.416 & Collahuasi 37 & 4.294 \\
\hline Co37-m9-c & 7.685 .856 & 535.416 & Collahuasi 37 & 4.294 \\
\hline UJN-m1-a & 7.687.389 & 535.976 & Ujina 10 & 4.347 \\
\hline UJN-m2-b & 7.687 .506 & 535.898 & Ujina 10 & 4.347 \\
\hline UJN-m2-e & 7.687 .506 & 535.898 & Ujina 10 & 4.347 \\
\hline
\end{tabular}




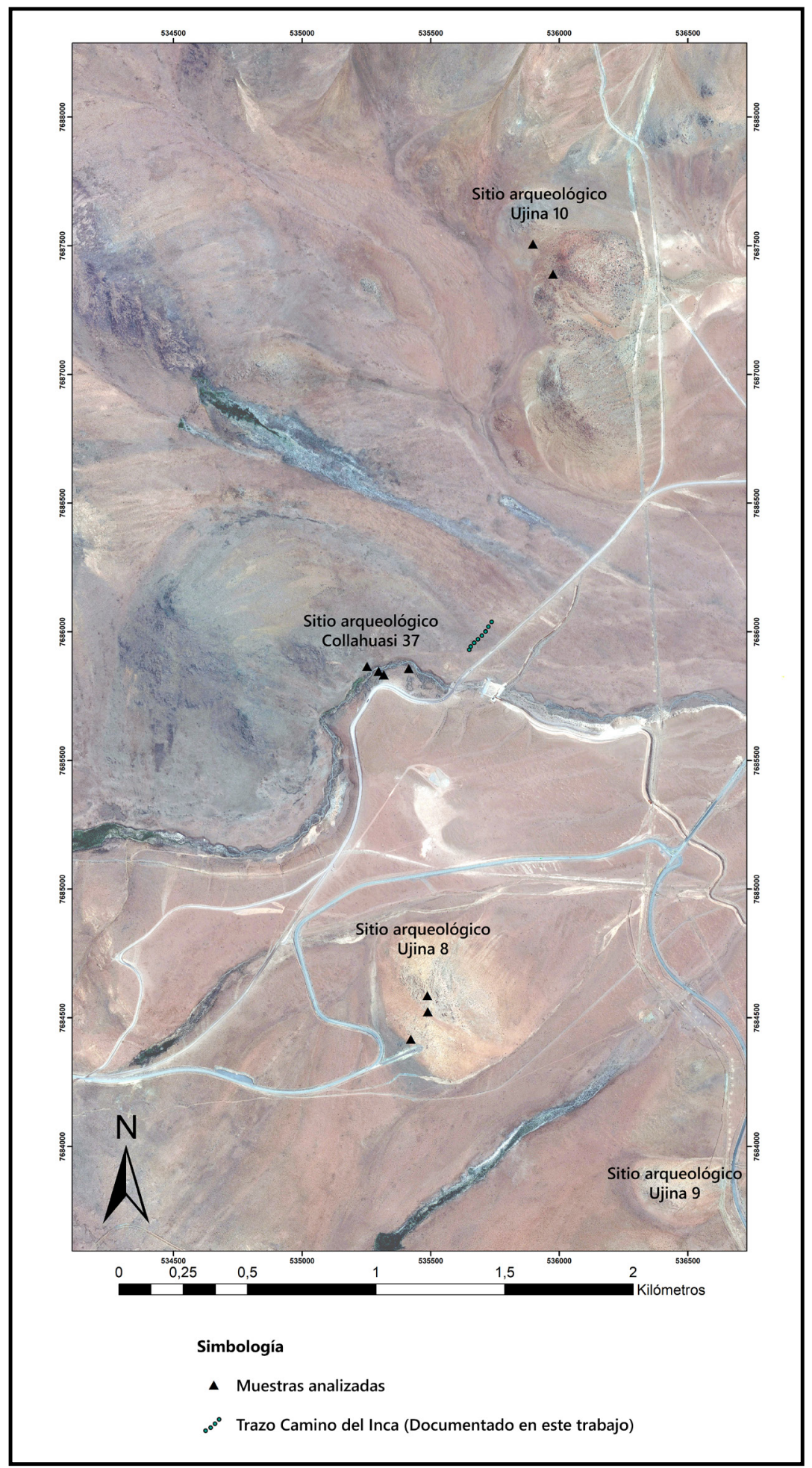

Figura 2. Proveniencia arqueológica de las muestras recolectadas. 


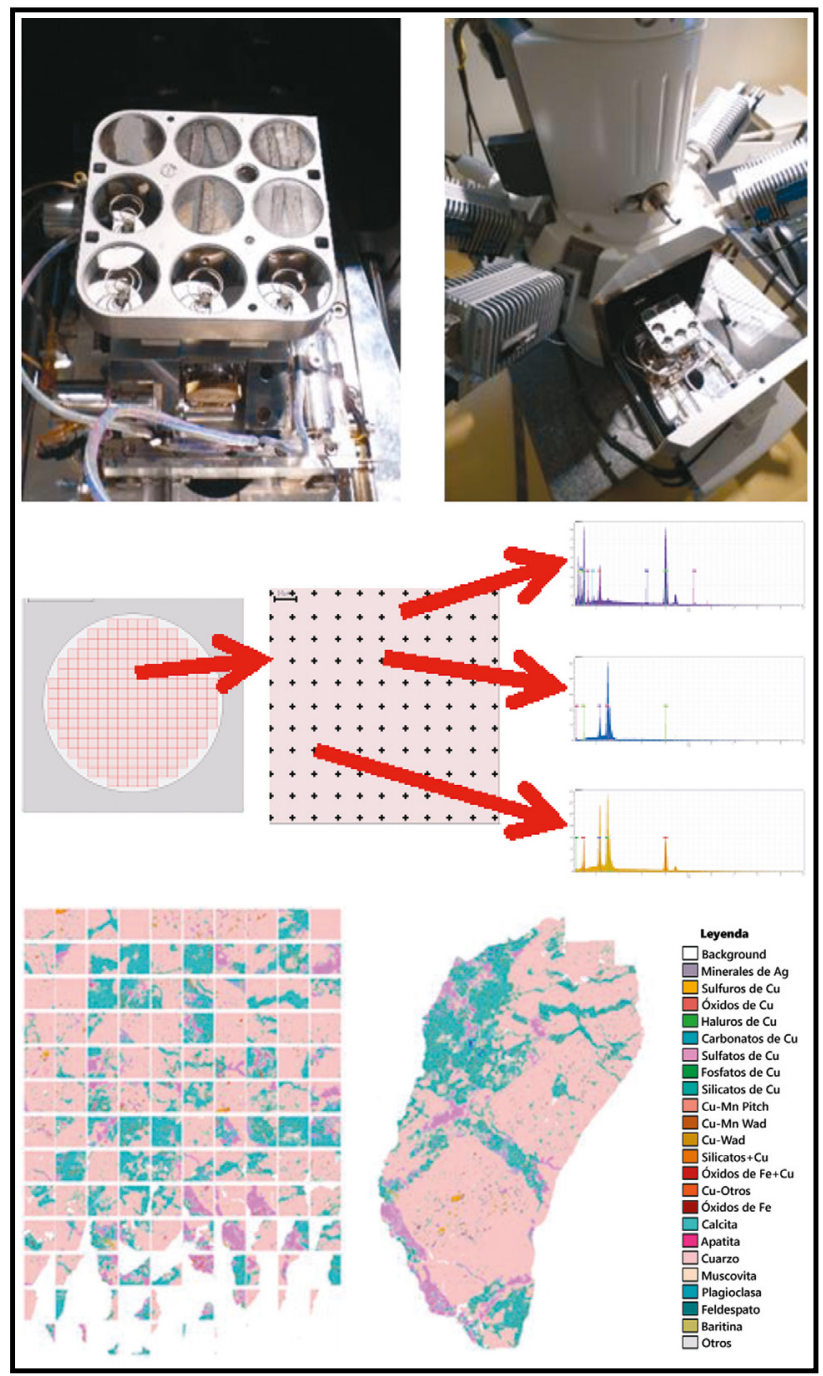

Figura 3. Diagrama esquemático que describe cómo QEMSCAN puede medir sistemáticamente a través del modo Field-Scan en toda el área de la muestra. Primera fila: imagen de las muestras montadas en resina, pulidas y cubiertas en carbón, ubicadas en un soporte de bloque para nueve muestras, a la espera de ser analizadas en el equipo ubicado en la Unidad de Equipamiento Científico-MAINI-UCN. Segunda fila: la briqueta pulida es separada en campos predefinidos.

Cada campo es sobrepuesto con una grilla predefinida de pixeles; el espectro de rayos X y el valor de retrodispersión obtenido para cada pixel son clasificados por la base de datos de identificación de minerales (SIP) y permiten la clasificación mineralógica y química. Tercera fila: se crea un mapa de color falso para cada campo, en el que cada color representa un grupo mineral o químico específico; los campos se combinan para generar un mosaico único del área analizada que se puede procesar e investigar con el software iDiscover. 


\section{Resultados}

\section{Mineralogía de las muestras}

La mineralogía modal se muestra gráficamente en la Figura 4 y numéricamente en la Tabla 2. Las muestras contienen una mezcla de mineralizaciones de cobre y minerales de ganga (por ejemplo, silicatos). La mineralización de Cu varía entre 19\% (Co37-m10-g) y
99\% (UJ8-m6-a). Otras mineralogías son variables, con algunas muestras que contienen óxidos de $\mathrm{Fe}$ (por ejemplo, casi el 20\% en Co37-m13-b), mientras que otras contienen abundantes minerales de As (por ejemplo, UJ8-m8-c, que tiene 36,58\%). Todas las muestras presentan niveles de $\mathrm{Ag}$ por encima de los límites de detección, que van desde $0,01 \%$ hasta $1,68 \%$ (Co37-m11-b), pero solo cinco muestras contienen niveles más altos $(>0,30 \%)$.
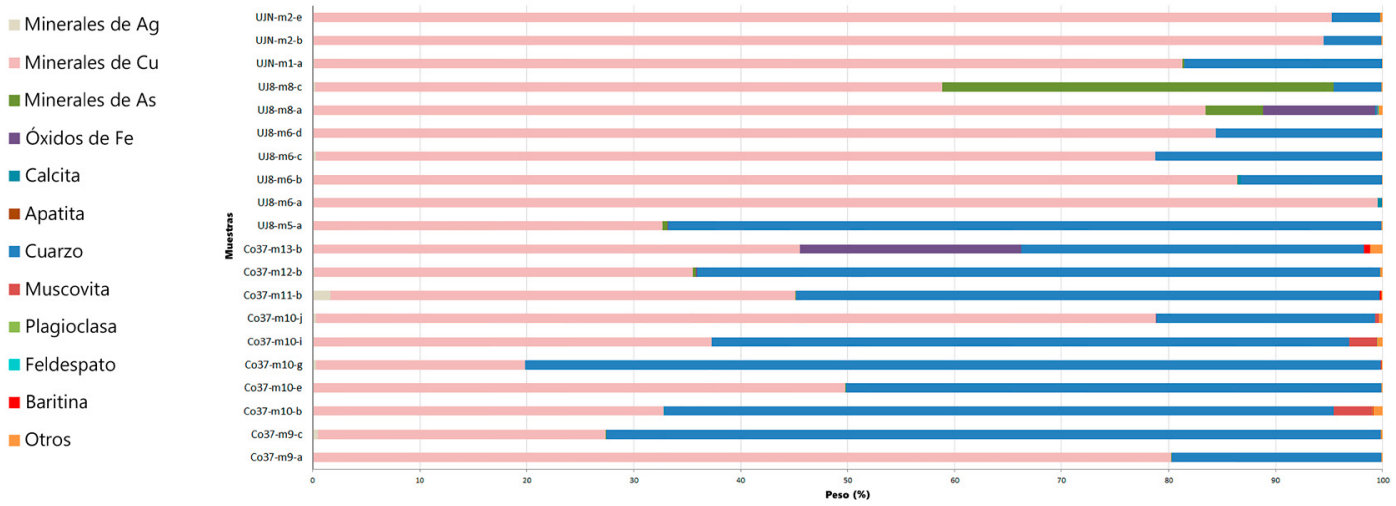

Figura 4. Análisis QEMSCAN de mineralogía modal: agrupados por minerales que contienen Cu y minerales de ganga.

Tabla 2. Mineralogía modal QEMSCAN: agrupada por grupos minerales que contienen $\mathrm{Cu}$ y minerales de ganga.

\begin{tabular}{|l|c|c|c|c|c|c|c|c|c|c|}
\hline & \multicolumn{7}{|c|}{ Muestras } \\
\cline { 2 - 12 } & $\begin{array}{c}\text { UJN- } \\
\mathbf{m} 2-\mathbf{c}\end{array}$ & $\begin{array}{c}\text { UJN- } \\
\mathbf{m 2 - b}\end{array}$ & $\begin{array}{c}\text { UJN- } \\
\mathbf{m 1 - a}\end{array}$ & $\begin{array}{c}\text { UJ8- } \\
\mathbf{m 8 - c}\end{array}$ & $\begin{array}{c}\text { UJ8- } \\
\mathbf{m 8 - a}\end{array}$ & $\begin{array}{c}\text { UJ8- } \\
\mathbf{m 6 - d}\end{array}$ & $\begin{array}{c}\text { UJ8- } \\
\mathbf{m 6 - c}\end{array}$ & $\begin{array}{c}\text { UJ8- } \\
\mathbf{m 6 - b}\end{array}$ & $\begin{array}{c}\text { UJ8- } \\
\mathbf{m 6 - a}\end{array}$ & $\begin{array}{c}\text { UJ8- } \\
\mathbf{m 5 - a}\end{array}$ \\
\hline Minerales de Ag & 0,00 & 0,04 & 0,13 & 0,23 & 0,04 & 0,02 & 0,31 & 0,02 & 0,06 & 0,11 \\
\hline Minerales de Cu & 95,27 & 94,50 & 81,19 & 58,63 & 83,41 & 84,44 & 78,45 & 86,40 & 99,49 & 32,61 \\
\hline Minerales de As & 0,00 & 0,00 & 0,14 & 36,58 & 5,40 & 0,00 & 0,00 & 0,02 & 0,00 & 0,46 \\
\hline Óxidos de Fe & 0,01 & 0,01 & 0,00 & 0,03 & 10,48 & 0,00 & 0,00 & 0,00 & 0,00 & 0,04 \\
\hline Calcita & 0,00 & 0,00 & 0,05 & 0,00 & 0,02 & 0,00 & 0,00 & 0,30 & 0,34 & 0,00 \\
\hline Apatita & 0,00 & 0,00 & 0,00 & 0,00 & 0,00 & 0,00 & 0,00 & 0,00 & 0,00 & 0,00 \\
\hline Cuarzo & 4,49 & 5,37 & 18,44 & 4,45 & 0,14 & 15,48 & 21,20 & 13,20 & 0,05 & 66,66 \\
\hline Muscovita & 0,00 & 0,00 & 0,00 & 0,00 & 0,04 & 0,00 & 0,00 & 0,00 & 0,00 & 0,00 \\
\hline Plagioclasa & 0,00 & 0,00 & 0,00 & 0,00 & 0,04 & 0,00 & 0,00 & 0,00 & 0,01 & 0,01 \\
\hline Feldespato & 0,00 & 0,00 & 0,00 & 0,00 & 0,03 & 0,00 & 0,00 & 0,01 & 0,00 & 0,00 \\
\hline Baritina & 0,00 & 0,02 & 0,00 & 0,00 & 0,00 & 0,00 & 0,00 & 0,00 & 0,00 & 0,01 \\
\hline Otros & 0,21 & 0,08 & 0,05 & 0,08 & 0,39 & 0,05 & 0,03 & 0,05 & 0,04 & 0,1 \\
\hline
\end{tabular}


Tabla 2. Mineralogía modal QEMSCAN: agrupada por grupos minerales que contienen Cu y minerales de ganga, continuación

\begin{tabular}{|c|c|c|c|c|c|c|c|c|c|c|}
\hline & \multicolumn{10}{|c|}{ Muestras } \\
\hline & $\begin{array}{l}\text { Co37- } \\
\text { m13-b }\end{array}$ & $\begin{array}{l}\text { Co37- } \\
\text { m12-b }\end{array}$ & $\begin{array}{l}\text { Co37- } \\
\text { m11-b }\end{array}$ & $\begin{array}{l}\text { Co37- } \\
\text { m10-j }\end{array}$ & $\begin{array}{l}\text { Co37- } \\
\text { m10-i }\end{array}$ & $\begin{array}{l}\text { Co37- } \\
\text { m10-g }\end{array}$ & $\begin{array}{l}\text { Co37- } \\
\text { m10-e }\end{array}$ & $\begin{array}{l}\text { Co37- } \\
\text { m10-b }\end{array}$ & $\begin{array}{l}\text { Co37- } \\
\text { m9-c }\end{array}$ & $\begin{array}{c}\text { Co37- } \\
\text { m9-a }\end{array}$ \\
\hline Minerales de Ag & 0,02 & 0,13 & 1,68 & 0,33 & 0,01 & 0,30 & 0,01 & 0,01 & 0,50 & 0,04 \\
\hline Minerales de Cu & 45,52 & 35,42 & 43,45 & 78,51 & 37,26 & 19,53 & 49,77 & 32,79 & 26,90 & 80,24 \\
\hline Minerales de As & 0,00 & 0,27 & 0,08 & 0,00 & 0,00 & 0,00 & 0,08 & 0,00 & 0,01 & 0,00 \\
\hline Óxidos de Fe & 20,71 & 0,11 & 0,00 & 0,00 & 0,02 & 0,00 & 0,00 & 0,01 & 0,00 & 0,02 \\
\hline Calcita & 0,00 & 0,00 & 0,00 & 0,00 & 0,00 & 0,00 & 0,02 & 0,04 & 0,00 & 0,01 \\
\hline Apatita & 0,00 & 0,00 & 0,00 & 0,00 & 0,00 & 0,00 & 0,00 & 0,00 & 0,00 & 0,00 \\
\hline Cuarzo & 32,00 & 63,85 & 54,54 & 20,50 & 59,58 & 80,03 & 50,03 & 62,58 & 72,42 & 19,59 \\
\hline Muscovita & 0,00 & 0,00 & 0,00 & 0,30 & 2,65 & 0,00 & 0,00 & 3,62 & 0,00 & 0,00 \\
\hline Plagioclasa & 0,01 & 0,00 & 0,00 & 0,01 & 0,00 & 0,01 & 0,00 & 0,01 & 0,01 & 0,00 \\
\hline Feldespato & 0,01 & 0,00 & 0,00 & 0,02 & 0,03 & 0,00 & 0,00 & 0,04 & 0,00 & 0,00 \\
\hline Baritina & 0,57 & 0,00 & 0,15 & 0,00 & 0,00 & 0,01 & 0,00 & 0,02 & 0,02 & 0,00 \\
\hline Otros & 1,2 & 0,2 & 0,1 & 0,3 & 0,4 & 0,1 & 0,1 & 0,9 & 0,1 & 0,1 \\
\hline
\end{tabular}

\section{Mineralización de $\mathrm{Cu}$}

Los grupos primarios de minerales de cobre identificados son sulfatos de $\mathrm{Cu}$, carbonatos de $\mathrm{Cu}$ y silicatos de $\mathrm{Cu}$, que varían entre cada una de las muestras individuales (Figura 5, Tabla 3). Otra mineralogía de $\mathrm{Cu}$ que se presenta en una abundancia modal más baja incluye sulfuros de $\mathrm{Cu}$, óxidos de $\mathrm{Cu}$, haluros de $\mathrm{Cu}$ y fosfatos de $\mathrm{Cu}$.

En el grupo de los sulfatos de $\mathrm{Cu}$, el mineral predominante es la brocantita $\left(\mathrm{Cu}_{4}\left(\mathrm{SO}_{4}\right)(\mathrm{OH})_{6}\right)$, y es la principal mineralización de las muestras UJ8-m8-c, UJ8-m6-c, Co37-m10-j, UJN-m2-e y UJN-m2-b, con porcentajes modales que van desde $-50-90 \%$. Además, los sulfatos de $\mathrm{Cu}$ tienen concentraciones intermedias que oscilan entre $-7-30 \%$ en las muestras Co37-m11-b, UJ8-m6-d, Co37-m10-i, Co37-m10-b, UJ8-m8-a, y UJ8-m5-a. Las otras muestras tienen $\leq 1 \%$ de sulfatos de $\mathrm{Cu}$.

En el grupo de los carbonatos de $\mathrm{Cu}$, el mineral predominante es la malaquita $\left(\mathrm{Cu}_{2}\left(\mathrm{CO}_{3}\right)(\mathrm{OH})_{2}\right)$, y es la principal mineralización de las muestras UJ8-m6b, Co37-m9-a, UJ8-m6-d, UJN-m1-a y UJ8-m6-a, con porcentajes modales de entre $-32-65 \%$. Ade- más, los carbonatos de $\mathrm{Cu}$ oscilan entre $-8-23 \%$ en las muestras Co37-m10-g, Co37-m9-c, Co37-m12b, Co37-m10-e, Co37-m13-b y Co37-m11-b. Las otras muestras presentan concentraciones $\leq 1 \%$ de malaquita.

En el grupo de los silicatos de $\mathrm{Cu}$, el mineral predominante es la crisocola $\left(\mathrm{Cu}_{2-\mathrm{x}} \mathrm{Al}_{\mathrm{x}}\left(\mathrm{H}_{2-\mathrm{x}} \mathrm{Si}_{2} \mathrm{O}_{5}\right)(\mathrm{OH})_{4}\right.$ $\cdot \mathrm{nH}_{2} \mathrm{O}(\mathrm{x}<1)$ ) (Figura 6), pero este grupo también incorpora copper pitch (silicato de cobre negro), con variaciones muy altas de $\mathrm{Cu}$ y $\mathrm{Si}$. La crisocola es el mineral principal en las muestras UJ8-m6-c, Co37-m9-a, Co37-m10-e y UJ8-m6-b, con porcentajes modales entre $-23-45 \%$. Se observaron concentraciones intermedias entre $-3-15 \%$ en las muestras Co37-m10-i, Co37-m10-j, Co37-m10-b, Co37-m13-b, Co37-m10-g, UJ8-m6-d, UJN-m1a, UJ8-m6-a, Co37-m9-c, y Co37-m12-b. Las muestras restantes tienen porcentajes $<2 \%$.

El grupo de los sulfuros de Cu está ausente en casi todas las muestras, o se presenta en niveles menores o traza, con excepción de la muestra Co37-m11-b que presenta $0,28 \%$ de sulfuros de $\mathrm{Cu}$, compuestos por calcopirita $\left(\mathrm{CuFeS}_{2}\right)$ y sulfosales de $\mathrm{Cu}$-Ag (p.e. Polibasita $\left.\left[(\mathrm{Ag}, \mathrm{Cu})_{6}(\mathrm{Sb}, \mathrm{As})_{2} \mathrm{~S}_{7}\right]\left[\mathrm{Ag}_{9} \mathrm{CuS}_{4}\right]\right)$. 
El grupo de los óxidos de $\mathrm{Cu}$ está presente en niveles bajos en todas las muestras, usualmente $<2 \%$, excepto en la muestra UJ8-m8-a que presenta un $10,27 \%$, predominantemente de especies minerales que tienen diferentes composiciones de óxidos de $\mathrm{Cu}-\mathrm{Fe}$. La mineralización de los óxidos de $\mathrm{Fe}-\mathrm{Cu}$ es muy variable. Cuando las concentraciones de $\mathrm{Cu}$ son inferiores al $10 \%$, la clasificación se encuentra dentro de los grupos de óxidos de $\mathrm{Fe}$, lo que produce varios porcentajes modales, por ejemplo UJ8-m8-a (43,03\%), seguido de Co37-m13-b (10,61\%) y Co37 -m12-b (1,38\%).
Las otras muestras tienen concentraciones modales $<1 \%$.

El grupo de los haluros de Cu está ausente o en niveles trazas $(<0,1 \%)$ en todas las muestras, con la excepción de tres de ellas: Co37-m9-a, Co37-m10-i, y Co37-m10-g con abundancias modales entre $-0,1$ a $0,3 \%$. El mineral principal en este grupo es la atacamita $\left(\mathrm{Cu}_{2}(\mathrm{OH})_{3} \mathrm{Cl}\right)$.

Los fosfatos de Cu están ausentes o en niveles trazas $(<0,1 \%)$ en todas las muestras.
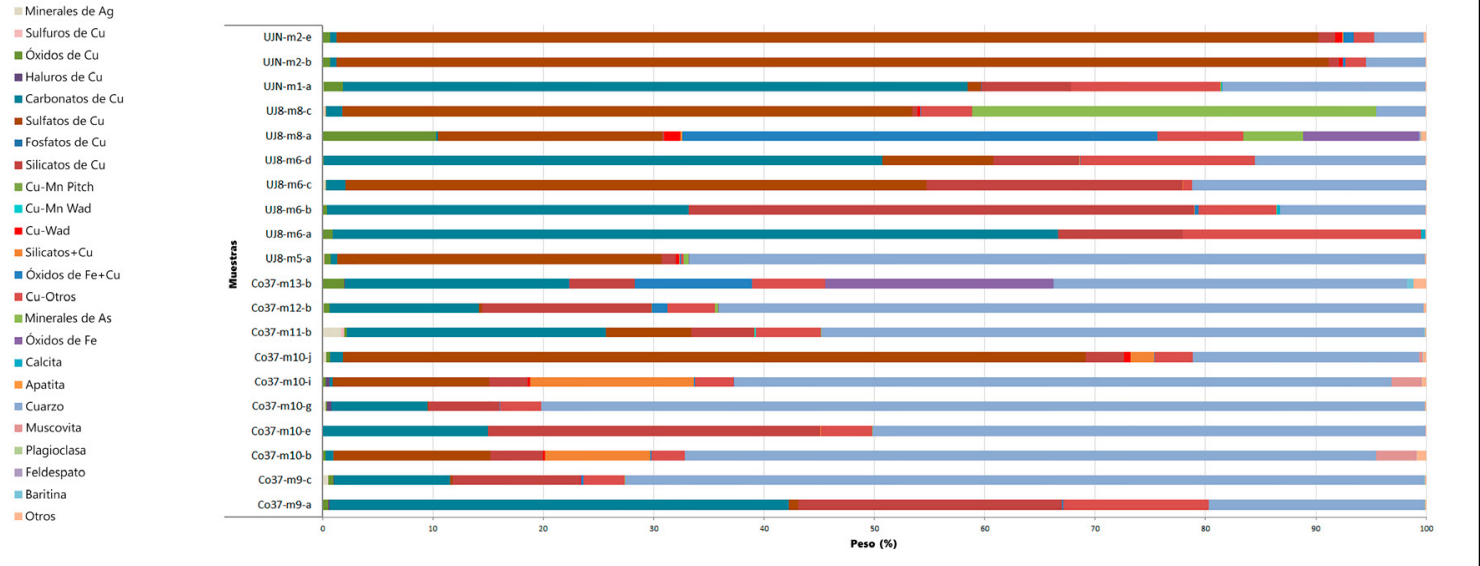

Figura 5. Análisis QEMSCAN de mineralogía modal: grupos primarios de minerales con contenidos de Cu. 
Tabla 3. Mineralogía modal QEMSCAN: comportamiento por grupos minerales que contienen Cu.

\begin{tabular}{|c|c|c|c|c|c|c|c|c|c|c|}
\hline & \multicolumn{10}{|c|}{ Muestras } \\
\hline & $\begin{array}{l}\text { UJN- } \\
\text { m2-e }\end{array}$ & $\begin{array}{l}\text { UJN- } \\
\text { m2-b }\end{array}$ & $\begin{array}{l}\text { UJN- } \\
\text { m1-a }\end{array}$ & $\begin{array}{l}\text { UJ8- } \\
\text { m8-c }\end{array}$ & $\begin{array}{l}\text { UJ8- } \\
\text { m8-a }\end{array}$ & $\begin{array}{l}\text { UJ8- } \\
\text { m6-d }\end{array}$ & $\begin{array}{l}\text { UJ8- } \\
\text { m6-c }\end{array}$ & $\begin{array}{l}\text { UJ8- } \\
\text { m6-b }\end{array}$ & $\begin{array}{l}\text { UJ8- } \\
\text { m6-a }\end{array}$ & $\begin{array}{l}\text { UJ8- } \\
\text { m5-a }\end{array}$ \\
\hline Minerales de Ag & 0,00 & 0,04 & 0,13 & 0,23 & 0,04 & 0,02 & 0,31 & 0,02 & 0,06 & 0,11 \\
\hline Sulfuros de Cu & 0,00 & 0,00 & 0,00 & 0,03 & 0,00 & 0,00 & 0,00 & 0,00 & 0,00 & 0,07 \\
\hline Óxidos de Cu & 0,67 & 0,66 & 1,74 & 0,07 & 10,27 & 0,11 & 0,04 & 0,41 & 0,90 & 0,60 \\
\hline Haluros de $\mathrm{Cu}$ & 0,00 & 0,00 & 0,00 & 0,00 & 0,00 & 0,00 & 0,00 & 0,00 & 0,00 & 0,00 \\
\hline Carbonatos de $\mathrm{Cu}$ & 0,59 & 0,60 & 56,59 & 1,47 & 0,18 & 50,62 & 1,75 & 32,77 & 65,70 & 0,54 \\
\hline Sulfatos de Cu & 88,98 & 89,85 & 1,21 & 51,62 & 20,28 & 10,02 & 52,64 & 0,00 & 0,01 & 29,39 \\
\hline Fosfatos de Cu & 0,00 & 0,00 & 0,04 & 0,00 & 0,00 & 0,00 & 0,01 & 0,00 & 0,00 & 0,00 \\
\hline Silicatos de Cu & 1,51 & 0,92 & 8,09 & 0,49 & 0,20 & 7,79 & 23,19 & 45,79 & 11,24 & 1,31 \\
\hline Cu-Mn Pitch & 0,00 & 0,00 & 0,00 & 0,00 & 0,00 & 0,01 & 0,00 & 0,00 & 0,00 & 0,00 \\
\hline Cu-Mn Wad & 0,00 & 0,00 & 0,00 & 0,00 & 0,00 & 0,00 & 0,00 & 0,00 & 0,00 & 0,00 \\
\hline Cu-Wad & 0,65 & 0,34 & 0,00 & 0,23 & 1,46 & 0,01 & 0,00 & 0,00 & 0,00 & 0,30 \\
\hline Silicatos $+\mathrm{Cu}$ & 0,07 & 0,00 & 0,01 & 0,01 & 0,20 & 0,03 & 0,01 & 0,02 & 0,01 & 0,01 \\
\hline Óxidos de $\mathrm{Fe}+\mathrm{Cu}$ & 0,97 & 0,23 & 0,01 & 0,07 & 43,03 & 0,04 & 0,00 & 0,33 & 0,01 & 0,16 \\
\hline Cu-Otros & 1,83 & 1,88 & 13,50 & 4,62 & 7,79 & 15,81 & 0,81 & 7,08 & 21,63 & 0,22 \\
\hline Minerales de As & 0,00 & 0,00 & 0,14 & 36,58 & 5,40 & 0,00 & 0,00 & 0,02 & 0,00 & 0,46 \\
\hline Óxidos de Fe & 0,01 & 0,01 & 0,00 & 0,03 & 10,48 & 0,00 & 0,00 & 0,00 & 0,00 & 0,04 \\
\hline Calcita & 0,00 & 0,00 & 0,05 & 0,00 & 0,02 & 0,00 & 0,00 & 0,30 & 0,34 & 0,00 \\
\hline Apatita & 0,00 & 0,00 & 0,00 & 0,00 & 0,00 & 0,00 & 0,00 & 0,00 & 0,00 & 0,00 \\
\hline Cuarzo & 4,49 & 5,37 & 18,44 & 4,45 & 0,14 & 15,48 & 21,20 & 13,20 & 0,05 & 66,66 \\
\hline Muscovita & 0,00 & 0,00 & 0,00 & 0,00 & 0,04 & 0,00 & 0,00 & 0,00 & 0,00 & 0,00 \\
\hline Plagioclasa & 0,00 & 0,00 & 0,00 & 0,00 & 0,04 & 0,00 & 0,00 & 0,00 & 0,01 & 0,01 \\
\hline Feldespato & 0,00 & 0,00 & 0,00 & 0,00 & 0,03 & 0,00 & 0,00 & 0,01 & 0,00 & 0,00 \\
\hline Baritina & 0,00 & 0,02 & 0,00 & 0,00 & 0,00 & 0,00 & 0,00 & 0,00 & 0,00 & 0,01 \\
\hline Otros & 0,21 & 0,08 & 0,05 & 0,08 & 0,39 & 0,05 & 0,03 & 0,05 & 0,04 & 0,10 \\
\hline
\end{tabular}


Tabla 3. Mineralogía modal QEMSCAN: comportamiento por grupos minerales que contienen $\mathrm{Cu}$, continuación.

\begin{tabular}{|c|c|c|c|c|c|c|c|c|c|c|}
\hline & \multicolumn{10}{|c|}{ Muestra } \\
\hline & $\begin{array}{l}\text { Co37- } \\
\text { m13-b }\end{array}$ & $\begin{array}{l}\text { Co37- } \\
\text { m12-b }\end{array}$ & $\begin{array}{l}\text { Co37- } \\
\text { m11-b }\end{array}$ & $\begin{array}{l}\text { Co37- } \\
\text { m10-j }\end{array}$ & $\begin{array}{l}\text { Co37- } \\
\text { m10-i }\end{array}$ & $\begin{array}{l}\text { Co37- } \\
\text { m10-g }\end{array}$ & $\begin{array}{l}\text { Co37- } \\
\text { m10-e }\end{array}$ & $\begin{array}{l}\text { Co37- } \\
\text { m10-b }\end{array}$ & $\begin{array}{l}\text { Co37- } \\
\text { m9-c }\end{array}$ & $\begin{array}{c}\text { Co37- } \\
\text { m9-a }\end{array}$ \\
\hline Minerales de Ag & 0,02 & 0,13 & 1,68 & 0,33 & 0,01 & 0,30 & 0,01 & 0,01 & 0,50 & 0,04 \\
\hline Sulfuros de Cu & 0,00 & 0,00 & 0,28 & 0,00 & 0,00 & 0,00 & 0,00 & 0,00 & 0,00 & 0,00 \\
\hline Óxidos de Cu & 1,97 & 0,48 & 0,28 & 0,36 & 0,26 & 0,11 & 0,02 & 0,28 & 0,51 & 0,47 \\
\hline Haluros de Cu & 0,06 & 0,00 & 0,00 & 0,01 & 0,35 & 0,39 & 0,00 & 0,03 & 0,05 & 0,14 \\
\hline Carbonatos de $\mathrm{Cu}$ & 20,28 & 13,54 & 23,47 & 1,14 & 0,31 & 8,75 & 14,96 & 0,64 & 10,50 & 41,59 \\
\hline Sulfatos de Cu & 0,01 & 0,34 & 7,68 & 67,29 & 14,18 & 0,04 & 0,01 & 14,28 & 0,22 & 0,85 \\
\hline Fosfatos de Cu & 0,02 & 0,00 & 0,00 & 0,01 & 0,00 & 0,00 & 0,01 & 0,00 & 0,00 & 0,00 \\
\hline Silicatos de Cu & 5,91 & 15,31 & 5,72 & 3,50 & 3,48 & 6,42 & 30,10 & 4,73 & 11,58 & 23,88 \\
\hline Cu-Mn Pitch & 0,01 & 0,03 & 0,03 & 0,00 & 0,00 & 0,00 & 0,00 & 0,00 & 0,00 & 0,00 \\
\hline Cu-Mn Wad & 0,00 & 0,02 & 0,12 & 0,00 & 0,00 & 0,00 & 0,00 & 0,00 & 0,00 & 0,00 \\
\hline $\mathrm{Cu}-\mathrm{Wad}$ & 0,00 & 0,00 & 0,01 & 0,59 & 0,23 & 0,01 & 0,00 & 0,18 & 0,02 & 0,00 \\
\hline Silicatos $+\mathrm{Cu}$ & 0,04 & 0,01 & 0,00 & 2,16 & 14,81 & 0,01 & 0,02 & 9,53 & 0,02 & 0,02 \\
\hline Óxidos de $\mathrm{Fe}+\mathrm{Cu}$ & 10,61 & 1,38 & 0,00 & 0,06 & 0,13 & 0,06 & 0,00 & 0,14 & 0,22 & 0,11 \\
\hline Cu-Otros & 6,60 & 4,30 & 5,85 & 3,40 & 3,50 & 3,73 & 4,65 & 2,99 & 3,76 & 13,17 \\
\hline Minerales de As & 0,00 & 0,27 & 0,08 & 0,00 & 0,00 & 0,00 & 0,08 & 0,00 & 0,01 & 0,00 \\
\hline Óxidos de Fe & 20,71 & 0,11 & 0,00 & 0,00 & 0,02 & 0,00 & 0,00 & 0,01 & 0,00 & 0,02 \\
\hline Calcita & 0,00 & 0,00 & 0,00 & 0,00 & 0,00 & 0,00 & 0,02 & 0,04 & 0,00 & 0,01 \\
\hline Apatita & 0,00 & 0,00 & 0,00 & 0,00 & 0,00 & 0,00 & 0,00 & 0,00 & 0,00 & 0,00 \\
\hline Cuarzo & 32,00 & 63,85 & 54,54 & 20,50 & 59,58 & 80,03 & 50,03 & 62,58 & 72,42 & 19,59 \\
\hline Muscovita & 0,00 & 0,00 & 0,00 & 0,30 & 2,65 & 0,00 & 0,00 & 3,62 & 0,00 & 0,00 \\
\hline Plagioclasa & 0,01 & 0,00 & 0,00 & 0,01 & 0,00 & 0,01 & 0,00 & 0,01 & 0,01 & 0,00 \\
\hline Feldespato & 0,01 & 0,00 & 0,00 & 0,02 & 0,03 & 0,00 & 0,00 & 0,04 & 0,00 & 0,00 \\
\hline Baritina & 0,57 & 0,00 & 0,15 & 0,00 & 0,00 & 0,01 & 0,00 & 0,02 & 0,02 & 0,00 \\
\hline Otros & 1,15 & 0,21 & 0,10 & 0,32 & 0,44 & 0,12 & 0,08 & 0,87 & 0,14 & 0,09 \\
\hline
\end{tabular}




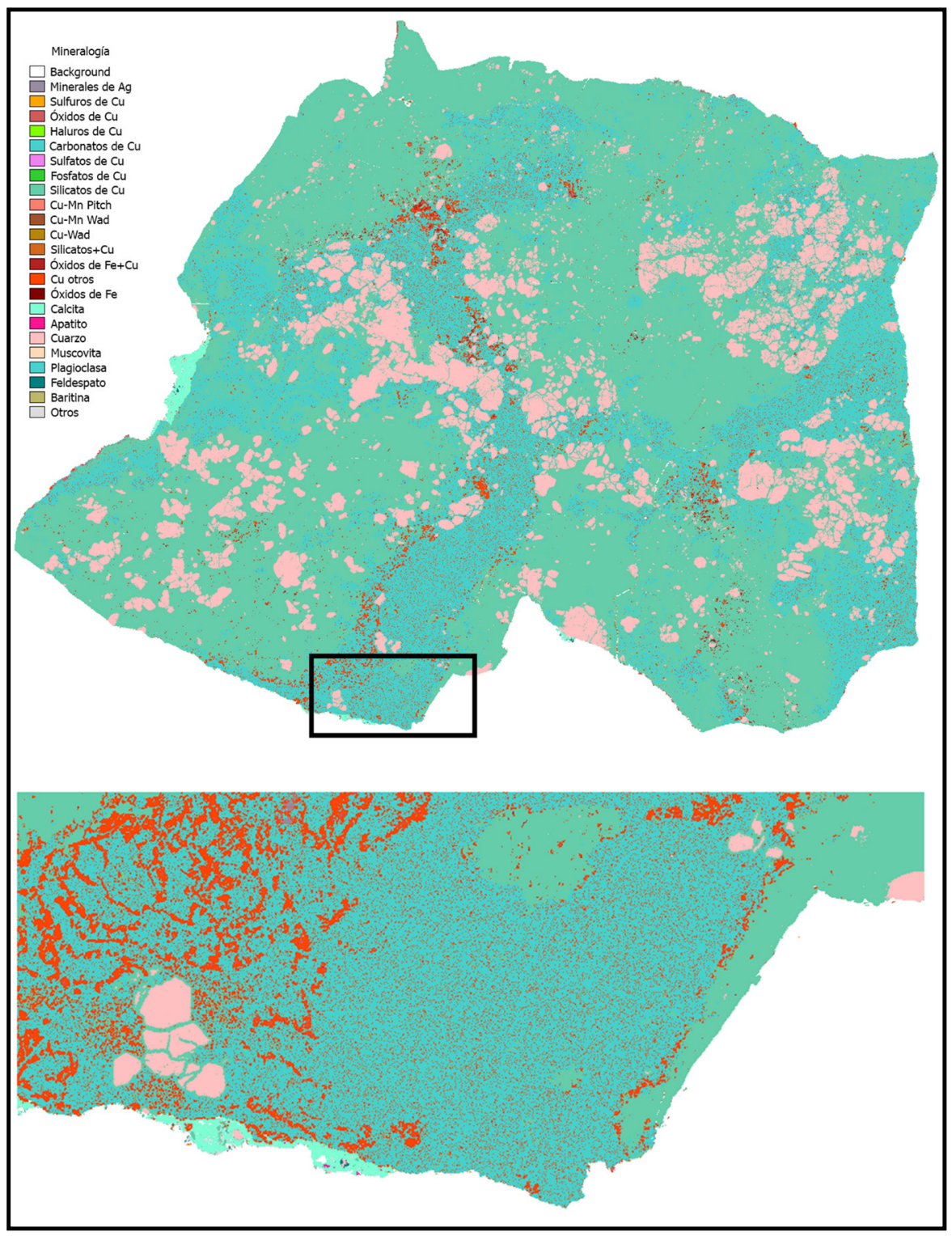

Figura 6. Resultados QEMSCAN para la muestra UJ8-m6-b que presenta altos niveles de crisocola (45,49\%) y malaquita $(32,77 \%)$.

\section{Mineralización de Ag}

Las muestras presentan mineralogía de plata en niveles trazas o no se detectan, con la excepción de cinco muestras que tienen una concentración relativamente alta que varía de 0,30 a $1,68 \%$. La muestra Co37-m11-b tiene el nivel más alto de mineralización de $\mathrm{Ag}(1,68 \%)$, mientras que las otras varían entre 0,3 y $0,5 \%$ en las muestras Co37-m9-c, Co37-m10-j, UJ8-m6-c y Co37-m10-g.
Una sulfosal de plata llamada acantita $\left(\mathrm{Ag}_{2} \mathrm{~S}\right)$ es el principal mineral de $\mathrm{Ag}$, sin embargo, también hay sulfatos de $\mathrm{Ag}-\mathrm{Cu}$ como estromeyerita $(\mathrm{CuAgS})$, mckinstryita $\left(\mathrm{Ag}_{5-\mathrm{x}} \mathrm{Cu}_{3+\mathrm{x}} \mathrm{S}_{4}(\mathrm{x}=0-0,28)\right.$ o jalpaita $\left(\mathrm{Ag}_{3} \mathrm{CuS}_{2}\right)$ (Figura 7), que se identificaron en las muestras Co37-m11-b, Co37-m12-b y UJ8-m6-b. Es importante mencionar que algunos sulfuros de $\mathrm{Cu}$, como la calcopirita, contienen niveles bajos de plata. 


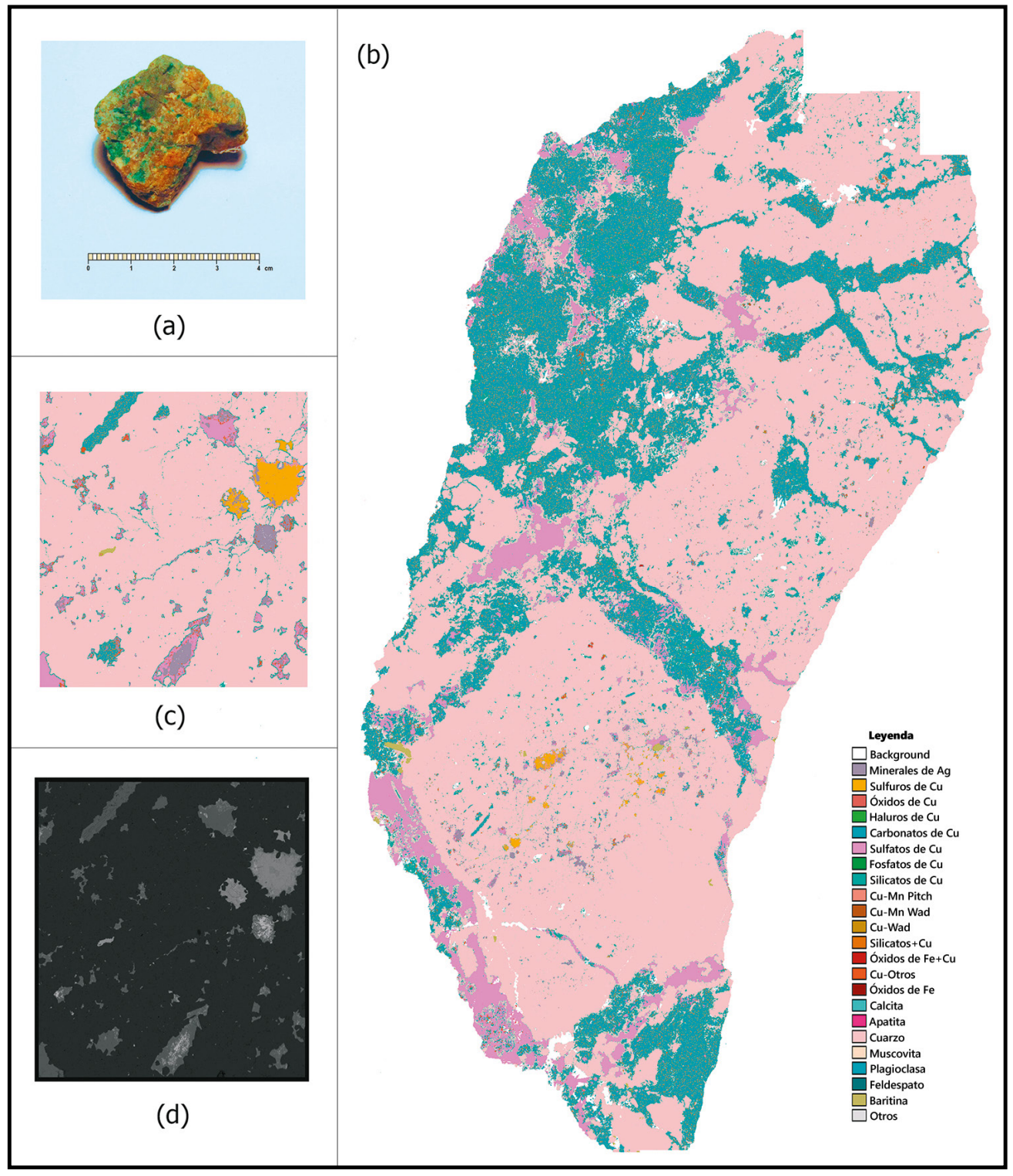

Figura 7. Resultados QEMSCAN para la muestra Co37-m11-b que presenta altos niveles de mineralogía de Ag (1,68 \%); (a) Fotografía macroscópica de la muestra Co37-m11-b; (b) Mapa mineralógico (muestra de aproximadamente $3 \mathrm{~cm}$ en su dimensión máxima); (c) Mapa mineral detallado (vista a escala de $1500 \mu \mathrm{m}$ );

(d) Imagen de retrodispersión de electrones (BSE). 


\section{Mineralización de As}

Los mapas elementales de arsénico indican que el As se incorpora predominantemente en una variedad de minerales como elemento menor o traza, como en los óxidos de Fe (Figura 8). La muestra con los niveles más altos de minerales que contie- nen As es UJ8-m8-c (36,58\%), que es extremadamente alta en comparación con las otras muestras, que tienen niveles mucho más bajos, por ejemplo, 5,40\% en UJ8-m8-a; 0,46\% en UJ8-m5-a; 0,27\% en Co37-m12-b; y 0,14\% en UJN-m1-a. Las otras muestras tienen niveles muy bajos o indetectables.

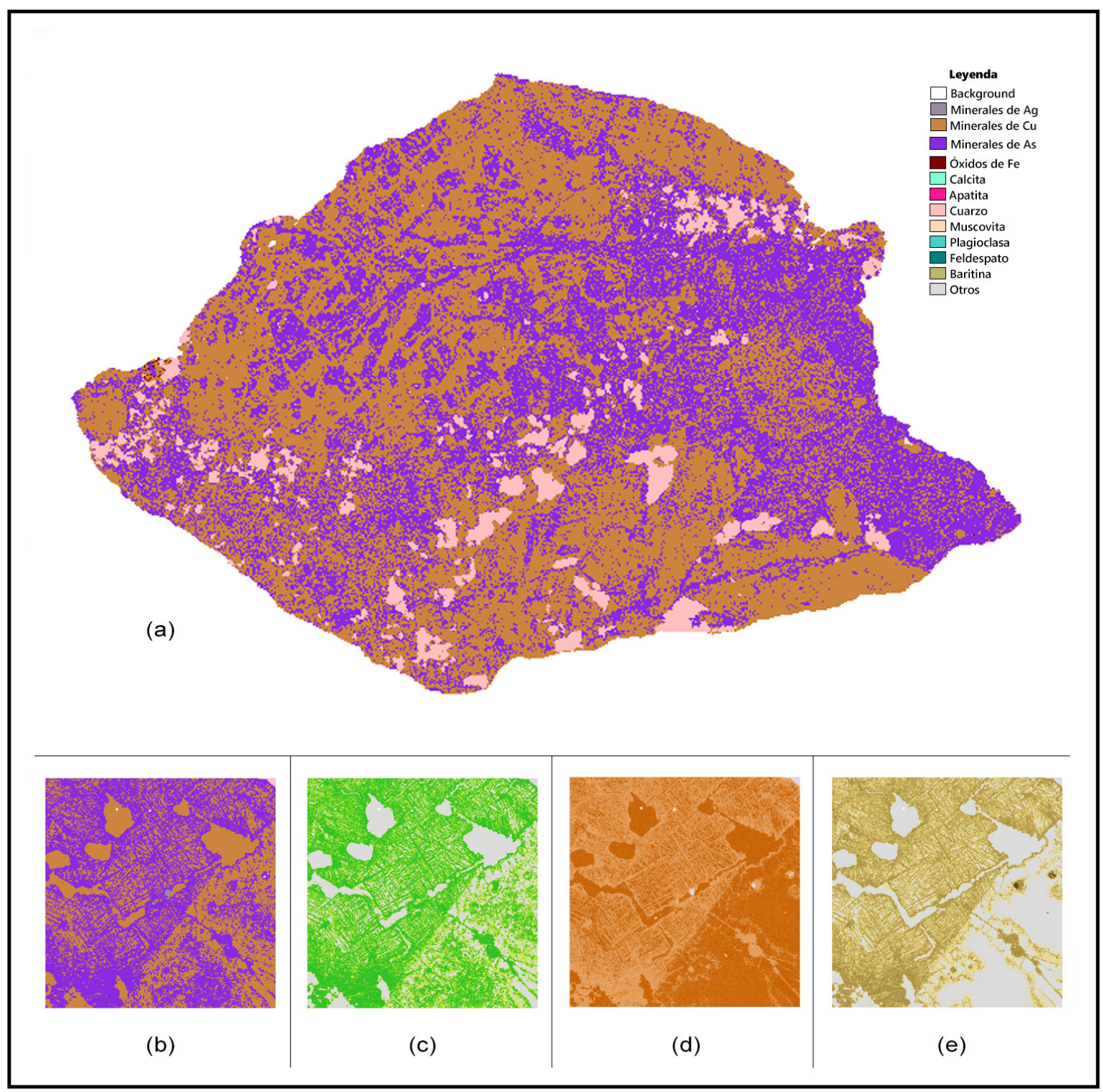

Figura 8. Los resultados QEMSCAN para la muestra UJ8-m8-c tienen los niveles más altos de minerales que contienen As $(36,58 \%)$, predominantemente incorporados en una variedad de minerales como elemento menor o traza, como los óxidos de Fe; (a) Mapa mineralógico; (b) Mapa mineral detallado (vista a escala de $1000 \mu \mathrm{m}$ ); (c) Mapa para el elemento As;

(d) Mapa para el elemento Cu (e) Mapa para el elemento Fe. 


\section{Mineralización de Fe}

Los mapas elementales de hierro indican que el Fe se incorpora predominantemente en minerales de $\mathrm{Cu}$, formando así una mezcla llamada óxidos de $\mathrm{Fe}+\mathrm{Cu}$ (Figura 9), así como de óxidos de Fe. Las dos muestras con los niveles más altos de $\mathrm{Fe}$, tanto como óxidos de $\mathrm{Fe}$ como óxidos de $\mathrm{Fe}+\mathrm{Cu}$ son Co37-m13-b
(10,6\% de óxidos de $\mathrm{Fe}+\mathrm{Cu}$ y 20,71\% de óxidos de $\mathrm{Fe}$ ), y UJ8-m8-a (43,0\% de óxidos de $\mathrm{Fe}+\mathrm{Cu}$ y $10,5 \%$ de óxidos de $\mathrm{Fe}$ ). Ambas muestras exhiben niveles muy altos de Fe en comparación con las otras muestras, que comúnmente se encuentran en niveles traza o no detectables. Por ejemplo, los óxidos de $\mathrm{Fe}+\mathrm{Cu}$ están presentes en nueve muestras por encima de $0,1 \%$, pero no exceden el $1 \%$.

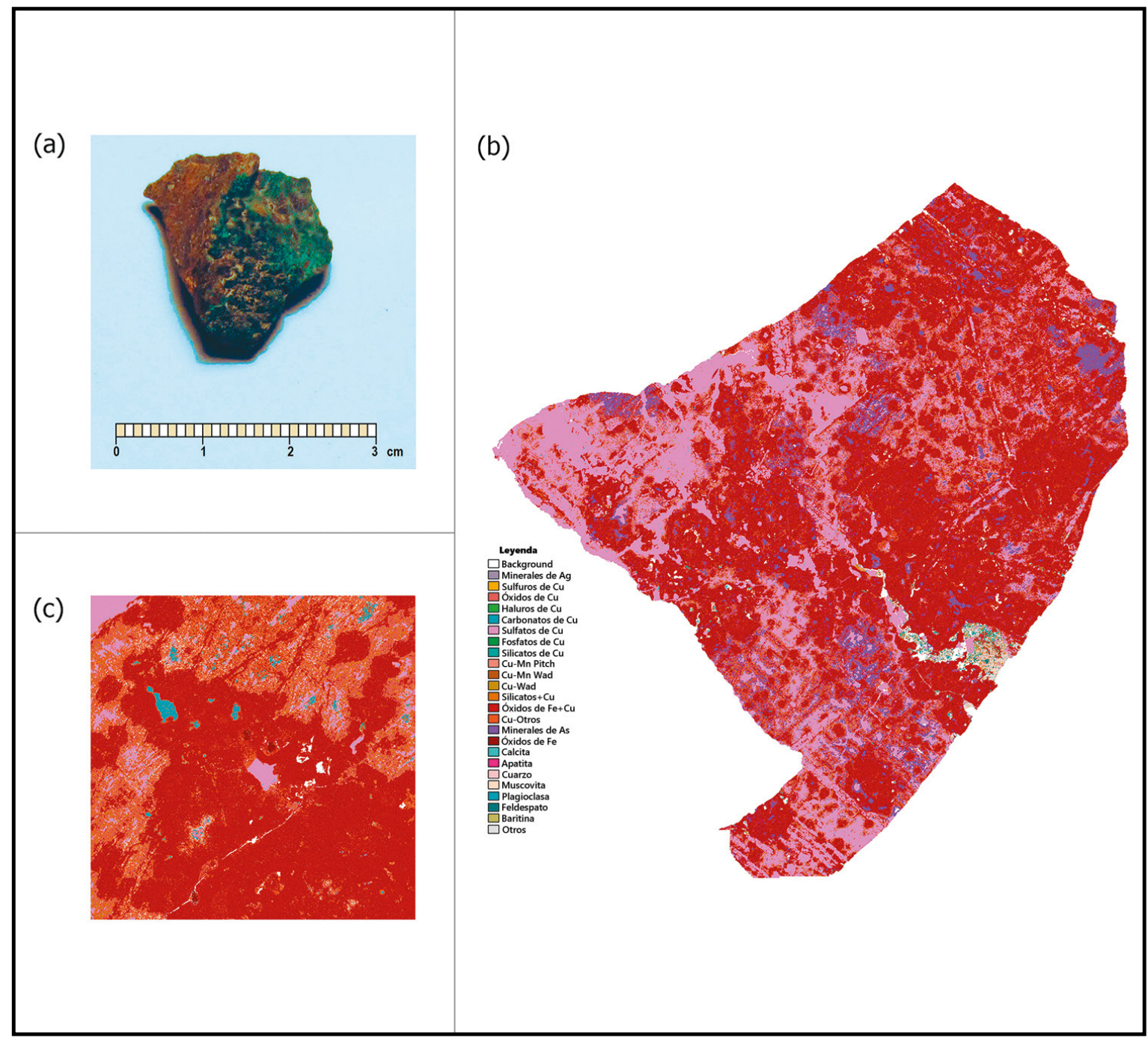

Figura 9. Resultados QEMSCAN para la muestra UJ8-m8-a que tiene los niveles más altos de $\mathrm{Fe}(43,0 \%$ óxidos de Fe+Cu y 10,5 \% óxidos de Fe); (a) fotografía macroscópica de muestra UJ8-m8-a; (b) Mapa mineralógico; (c) Mapa mineral detallado (vista a escala de $2500 \mu \mathrm{m}$ ). 


\section{Discusiones}

Se presume que las muestras analizadas son ejemplos de los minerales primarios utilizados directamente para la fundición prehispánica y, por lo tanto, representan los tipos de minerales de cobre utilizados en los hornos metalúrgicos de los sitios Ujina 8 y Ujina 10. Estas muestras son pequeñas, con un $50 \%$ de las muestras analizadas aproximadamente de $2 \mathrm{~cm}$ en su dimensión máxima, probablemente debido a que fueron recuperadas en los sitios de fundición habiendo ya pasado por un proceso de molienda, trituración y preselección antes de la fundición (Salazar et al., 2013a; Figueroa, 2015).

Los resultados indican que se usaron principalmente minerales oxidados de cobre en los hornos de fundición, correspondientes a brocantita, malaquita y crisocola. Esta asociación mineral está presente en la mayoría de las muestras estudiadas, y sugiere que los minerales seleccionados provienen de la misma fuente, dado que las muestras comparten la misma matriz silicatada. Además, la ganga en las muestras es casi exclusivamente cuarzo. Es importante tener en cuenta que estos resultados no representan el porcentaje de mineralización de la fuente, y solo pueden usarse como una guía para relacionar estos minerales con la fuente exacta de origen en el Distrito Collahuasi. Esto se debe a que las muestras se recogen a mano y son pequeñas y, por lo tanto, están sesgadas hacia la mineralización y no son comparables a las rocas de origen a una escala representativa más grande. Sin embargo, las especies minerales presentes en las muestras pueden usarse como un filtro comparativo para apoyar o rechazar posibles fuentes.

La relación mineral-ganga es variable, pero es notable que cuanto mayor sea el porcentaje de mineralización de cobre, menor es el porcentaje de ganga presente, reforzando la idea de preselección de muestras vinculadas al proceso de fundición para mejorar el resultado de la metalurgia, como se encuentra en los sitios de producción metalúrgica $U j$ jina 8 y Ujina 10.

Las muestras que tienen los porcentajes más altos de cuarzo (20-80\%) corresponden a las recogidas en el sitio Collahuasi 37, lo que podría indicar que la trituración y clasificación final de los minerales ocurrió en el sitio residencial de Collahuasi 37 y no en los sitios de fundición con alta exposición al viento. En Collahuasi 37 los metalurgistas habrían elegido las muestras con porcentajes más altos de cobre después de la trituración final, para trasladarlos a los sitios de producción metalúrgica, dejando en Collahuasi 37 desmontes con baja ley de cobre. Alternativamente, si el proceso de selección se produce en los sitios de fundición, los fragmentos de ganga se habrían transferido desde los sitios metalúrgicos al campamento habitacional, lo que parece menos probable. Sin embargo, no puede ignorarse la posibilidad de varias etapas de descarte de material en distintas etapas del proceso minero y metalúrgico, comenzando, por ejemplo, con un descarte primario en la mina, secundario en el chancado y hasta terciario en los hornos.

El contenido de cobre de los minerales presentes de mayor a menor nivel de abundancia de cobre son malaquita $(57,6 \% \mathrm{Cu})$, brocantita $(56,2 \% \mathrm{Cu})$ y crisocola $(36,2 \% \mathrm{Cu})$, respectivamente. En general, la malaquita y la crisocola se presentan con texturas simples, moteadas, esferulítica-concéntrica o con bandeamiento coloforme. Esto implicaría que los metalurgistas del Distrito Collahuasi recuperarían la mayor cantidad de cobre de la asociación mineral malaquita-brocantita. Sin embargo, los primeros resultados del estudio metalúrgico de las escorias de Collahuasi muestran que la fundición no se limitó a malaquita-brocantita, sino que también incluyó a la crisocola en gran parte (Mille et al., 2015; Gosselin, 2016). Las escorias se componen principalmente de una matriz vítrea silicatada, en la que cristalizaron algunas fases como wollastonita, piroxenos (hedenbergita, augita, diópsido) y óxidos de hierro (magnetita) y en las que también se pueden encontrar inclusiones de cobre en forma metálica. La presencia de algunos minerales neoformados, como la ortoclasa, confirma que se alcanzaron temperaturas muy altas en el proceso metalúrgico (Gosselin, 2016, Figueroa et al., 2018).

Las impurezas más importantes en los minerales de cobre identificadas en este estudio son As, Ag y Fe. Estos tipos de impurezas, presentes en la materia prima, implican que deben estar presentes también en el cobre metálico producido por la reducción de los minerales de cobre. En el sitio arqueológico de Miño, 
ubicado en las cercanías del Distrito Collahuasi, Salazar et al. (2013b) describen las impurezas Fe, Ag y As presentes en algunos desechos metalúrgicos (por ejemplo, escorias), lo que podría implicar que ambos sitios tenían acceso a las mismas fuentes minerales. Sin embargo, el tipo de metalurgia en Miño y Collahuasi no coinciden (Figueroa et al., 2015). Si bien comparten algunas características comunes con la metalurgia prehispánica de la vecina localidad de Mińo: las escorias son muy viscosas y gran parte del cobre metálico queda atrapado en la escoria, incluso bajo la forma de óxidos de cobre así como de prills de cobre; existen diferencias tales como el fundente de manganeso, sistemáticamente observado en este último sitio pero ausente en las escorias de Ujina 8. Además, las escorias de Ujina 8 presentan fragmentos de inclusiones de piedras no reducidas que corresponden ciertamente a fragmentos de las paredes de los hornos metalúrgicos y por su parte, los desechos de Miño demuestran que los hornos metalúrgicos utilizados fueron fabricados en arcilla. Es decir, se trata de hornos completamente diferentes (Mille, Figueroa, Salazar y Menzies, 2015). Además, hay evidencia de mineral rodeado de zonas oxidadas que contienen el arsenato de cobre: chenevixita $\left(\mathrm{Cu}_{2} \mathrm{Fe}_{2}\left(\mathrm{AsO}_{4}\right)_{2}(\mathrm{OH})_{2} \cdot \mathrm{H}_{2} \mathrm{O}\right)$, probablemente derivado de la enargita en el Distrito Collahuasi (Dick, Chávez, González y Bisso, 1994). Además, otros estudios indican que $\mathrm{Sb}$ es también una impureza importante en los productos de cobre metálico (Mille et al., 2015, Figueroa et al., 2018), sin embargo, no se observó mineralogía de $\mathrm{Sb}$ en las muestras de mineral. Esto no excluye la posibilidad de que el $\mathrm{Sb}$ se encuentre en niveles menores o mínimos en otros minerales dentro de estas muestras.

Finalmente, en los tres sitios arqueológicos estudiados, las muestras exhiben la misma asociación mineralógica de cobre predominante, es decir, malaquita-brocantitacrisocola. No es posible determinar cuál de estos minerales puede ser el predominante para cada sitio, ya que las muestras analizadas son fragmentos y no representan una veta o un contexto más general.

Sin embargo, las observaciones de piques mineros (históricos) cerca de los sitios (Figueroa et al., 2018) indican que la roca de caja es una andesita con alteración argílica. Además, se sugiere que la fuente de minerales utilizada por los antiguos metalurgistas podría ser muy similar a estos piques mineros encontrados, debido a que:

i) en el sitio Collahuasi 37, específicamente en la excavación de la estructura 15 asociada al sector E se observó macroscópicamente un fragmento de andesita, con fuerte alteración argílica y presencia de oxidados de cobre (arcillas de cobre, crisocola y brocantita) y,

ii) en el proceso de alteración hidrotermal, las reacciones de hidrólisis pueden alterar la plagioclasa (mineral esencial en una andesita) a cuarzo, de modo que este cuarzo como ganga en las muestras analizadas podría ser cuarzo secundario producto de la alteración de una andesita como roca de caja.

Por estos motivos, es muy importante comparar las muestras analizadas con aquellas encontradas en los piques mineros 1 y 2 (detallados en Figueroa et al., 2018), mediante análisis más específicos, por ejemplo, análisis isotópicos y de elementos trazas, para confirmar o excluir si esta puede ser la fuente del cobre. De lo contrario, se requeriría una búsqueda ampliada de fuentes alternativas en el área, como vetas de características similares. Un área preferencial que podría explorarse son los afloramientos de andesitas ubicadas al suroeste de los sitios arqueológicos.

\section{Conclusiones}

Si bien los estudios de procedencia de minerales de cobre presentan algunas vicisitudes estructurales (Bourgarit y Mille, 2014), en esta investigación hemos contribuido metodológicamente al desarrollo de este campo aplicado en materiales arqueológicos, a partir del estudio in fine de las primeras fases de la cadena operativa minero-metalúrgica.

El norte de Chile es conocido por ser un territorio de enormes concentraciones y diversidades de yacimientos cupríferos. No obstante, la expertise mineralógica desde las ciencias de la tierra producida por este commodity permite cada vez más encontrar soluciones precisas y adaptadas a las preguntas de investigación formuladas desde las ciencias sociales y los estudios patrimoniales. La incorporación de 
nuevas tecnologías no destructivas en el estudio de muestras arqueológicas prehispánicas de minerales del Distrito Collahuasi ha ayudado a obtener un nivel de información muy detallado. Específicamente, la mineralogía automatizada (QEMSCAN) arrojó resultados pormenorizados de la mineralogía de $\mathrm{Cu}$ utilizada como materia prima en el proceso de fundición de minerales de cobre en los hornos metalúrgicos prehispánicos del Distrito Collahuasi. Además, fue posible identificar la presencia y asociación mineralógica de impurezas como As, Ag y Fe. Finalmente, la descripción de estas muestras proporciona una referencia para la comparación con futuros estudios de otros sitios arqueológicos situados en las áreas cercanas a Collahuasi (p.e., Tarapacá, Altiplano Meridional, Loa, Lípez) con presencia de minerales o metales, de manera de ir configurando el entramado de la economía del cobre en los períodos prehispánicos tardíos.

\section{Agradecimientos}

Esta investigación fue apoyada por el Proyecto Fondecyt 11130651 "Tecnología y organización de la producción de cobre en el Distrito Collahuasi, altiplano sur de Tarapacá" y el Proyecto CNRS Laboratoire International Associé (LIA) Mines Atacama. A su vez, agradecemos al proyecto financiado por AGLAE (C2RMF) "2D Mapping of trace elements copper ores from pre-hispanic within northern Chile". Los análisis presentados se realizaron mediante QEMSCAN modelo E430 ubicado en la Unidad de Equipamiento Científico-MAINI-UCN, Antofagasta, Chile. Agradecemos a la Dirección General de Postgrado de UCN, quienes apoyaron este trabajo a través de FCAC 057/2019-UCN y a la Agencia Nacional de Investigación y Desarrollo (ANID)/BECA DOCTORADO NACIONAL/2018-21181070. Además, queremos agradecer al equipo de investigación, especialmente a: Ariadna Cifuentes, Hugo Carrión, Ignacia Corral, Paulina Corrales, Cristián González, Manon Gosselin, al Departamento de Medioambiente de Compañía Minera Doña Inés de Collahuasi. Finalmente a los organizadores del simposio XIII Reunión de Historiadores de la Minería Latinoamericana M. Florencia Becerra y Pablo Cruz, del cual forma parte este trabajo y a los revisores que realizaron un trabajo muy minucioso sobre el manuscrito.

\section{Referencias citadas}

Berenguer, J., Sanhueza, C. y Cáceres, I. (2011). Diagonales incaicas, interacción interregional y dominación en el altiplano de Tarapacá, norte de Chile. En Núñez, L. y Nielsen, A. (Eds.). En ruta, arqueologia, historia y etnografía del tráfico sur andino (pp. 247-283). Córdoba: Encuentro Grupo Editor.

Bourgarit, D. y Mille, B. (2014). Provenance du cuivre et alliages. Dillmann, Philippe; Bellot-Gurlet, Ludovic. Circulation et provenance des matériaux dans les sociétés anciennes. Editions des archives contemporaines, 103-130.

Campos, E., Menzies, A., Hernández V., Sola, S., Barraza, M. y Riquelme, R. (2015). Understanding Exotic-Cu Mineralisation: Part I - Characterisation of Chrysocolla, 13th SGA meeting, Nancy.

Dick, L. A., Chávez, W., González, A. y Bisso, C. (1994). Geologic setting and mineralogy of the Cu-Ag-(As) Rosario vein system, Collahuasi Distric, Chile. Society of Economic Geologists Newsletter, 19, 6-11.

Figueroa, V. (2015). Tecnología y organización de la producción de cobre en el Distrito Collahuasi, altiplano sur de Tarapacá. Informe Año 1, Proyecto Fondecyt N ${ }^{\circ}$ 11130651. Manuscrito en posesión del autor.

Figueroa, V. (2016). Tecnología y organización de la producción de cobre en el Distrito Collahuasi, altiplano sur de Tarapacá. Informe Año 2, Proyecto Fondecyt N ${ }^{\circ}$ 11130651. Manuscrito en posesión del autor.

Figueroa, V., Mille, B., Salazar, D., Berenguer, J., Menzies, A., Sapiains, P., Cifuentes, A., Joly, D. (2018). A new major Prehispanic copper production center identified at Collahuasi, southern Tarapacá Altiplano (Chile). Chungara. Revista de Antropología Chilena, 50(4), 557-575

Figueroa, V., Salazar, D., Mille, B., Berenguer, J., Cifuentes, A., Corrales, P., Menzies, A., Joly, D. y Sapiains, P. (2015). Tecnología y organización de la producción prehispánica del cobre en Collahuasi (Altiplano Meridional, Chile). En XX Congreso Nacional de Arqueología Chilena, Concepción, 5-9 October 2015.

Figueroa, V., Salazar, D., Salinas, H., Núñez-Regueiro, P. y Manríquez, G. (2013). Pre-Hispanic mining ergology of 
northern Chile: an archaeological perspective. Chungara. Revista de Antropología Chilena, 45(1), 61-81.

Gosselin, M. (2016). Study of pre-Hispanic extractive metallurgy process at Collahuasi (Chile). Mémoire de stage de 2e année de l'école nationale supérieure de chimie de Rennes, sous la direction de Benoît Mille et Laurent le Pollès, $53 \mathrm{pp}$.

Menzies, A., Campos, E., Hernández, V., Sola, S. y Riquelme, R. (2015a). Understanding Exotic-Cu Mineralisation: Part II - Characterisation of Black Copper ("Cobre Negro") ore, 13th SGA meeting, Nancy.

Menzies, A., Figueroa, V., Mille, B., Salazar, D., Wilke, H., Sapiains, P., Fonseca, P. y Ossandón, C. (2015b). Automated mineralogical analysis of archaeological samples from northern Chile: Case Study I - pre-Hispanic copper mining in the Collahuasi district, region Tarapacá. En XIV Congreso Geológico Chileno, Actas Vol. III, 367370, La Serena, 4-8 October 2015.

Menzies, A., Uribe, M., Erazo, F., Ossandón, C. y Fonseca, P. (2015c). Automated mineralogical analysis of archaeological samples from northern Chile: Case Study II - Ceramics. En XIV Congreso Geológico Chileno, Actas Vol. III, 371-374, La Serena, 4-8 October 2015.

Mille, B., Salazar, D., Bourgarit, D., Figueroa, V., Perlès, C. y Berenguer, J. (2013). Emergence of large scale copper production during the Early Bronze Age in Saint-Véran (France) and in Prehispanic Northern Chile: a comparative research program. The Crucible (Neswletter of the Historical Metallurgical Society), 84, 8-9.

Mille, B., Figueroa, V., Salazar, D. y Menzies, A. (2015). Technology and organisation of prehispanic copper production at Collahuasi (Southern Altiplano, Chile). En Archaeometallurgy in Europe, Madrid, 1-3 June 2015.

Rees, C. (1999). Elaboración, distribución y consumo de cuentas de malaquita y crisocola durante el Periodo Formativo en la Vega de Turi y sus inmediaciones, subregión del Río Salado, norte de Chile. En Aschero, C., Korstanje, A. y Vuoto, P. (Eds.). Los Tres Reinos: Prácticas de Recolección en el Cono Sur de América (pp. 85-98). Instituto de Arqueología y Museo, Universidad Nacional de Tucumán, Tucumán.
Romero, A. y Briones, L. (1999). CO-37: Estado y planificación inca en Collahuasi (Provincia de Iquique, I región, Chile). Estudios Atacameños, 18, 141-154.

Salazar, D. (2010). Tras la senda del cobre Atacameño: la historia minera de San José de El Abra. (2a ed.). SCM El Abra.

Salazar, D., Castro, V., Michelow, J., Salinas, H., Figueroa, V. y Mille, B. (2010a). Minería y metalurgia en la costa arreica de la región de Antofagasta, norte de Chile. Boletín del Museo Chileno de Arte Precolombino, 15(1), 9-23.

Salazar, D., Figueroa, V., Mille, B., Morata, D., Salinas, H. (2010b). Metalurgia prehispánica en las sociedades costeras del norte de Chile (Quebrada Mamilla, Tocopilla). Estudios Atacameños. Arqueología y Antropología Surandinas, 40, 23-42.

Salazar, D., Figueroa, V., Morata, D., Mille, B., Manríquez, G., Cifuentes, A. (2011). Metalurgia en San Pedro de Atacama durante el Período Medio: nuevos datos, nuevas preguntas. Revista de Antropología, 23, 123-148.

Salazar, D., Figueroa, V., Mille, B., Carrión, H., Balestro, F. y Berenguer, J. (2013a). Informe minero-metalúrgico: prospecciones, excavaciones y análisis. Informe Año 3, Proyecto Fondecyt N $\mathrm{N}^{\circ} 1100905$.

Salazar, D., Berenguer, J. y Vega, G. (2013b). Paisajes minero-metalúrgicos incaicos en Atacama y en el Altiplano Sur de Tarapacá (Norte de Chile). Chungara. Revista de Antropología Chilena, 45(1), 83-103.

Sapiains, P. (2016). Mineralogía de menas y su relación con la explotación prehispánica de cobre, Distrito Minero de Collahuasi, Región de Tarapacá, Chile. Memoria de Título (Inédito), Universidad Católica del Norte, Departamento de Ciencias Geológicas, 105 pp.

Sepúlveda, M., Figueroa, V., Pagés-Camagna, S. (2013). Copper pigment- making in the Atacama Desert (northern Chile). Latin American Antiquity, 24(4), 467-482.

Sepúlveda, M., Figueroa, V. y Cárcamo, J. (2014). Pigmentos y pinturas de mineral de Cobre en la región de Tarapacá, norte de Chile: Nuevos datos para una tecnología pigmentaria prehispánica. Estudios Atacameños. Arqueología y Antropología Surandinas, 48, 23-37. 
Sillitoe, R. H. (2005). Supergene oxidized and enriched porphyry copper and related deposits. Economic Geology, $100,723-768$.

Ward, I., Merigot, K. y McInnes, B. I. A. (2018) Application of Quantitative Mineralogical Analysis in archaeological micromorphology: a case study from Barrow Is., Western Australia. Journal of Archaeological Method and Theory, 25(1), 45-68.

(9) (1) Este es un artículo de acceso abierto bajo licencia Creative Commons Reconocimiento 4.0 Internacional 